\title{
Consistent Lorentz Violation in Flat and Curved Space
}

\author{
Gia Dvali, ${ }^{*}$ Oriol Pujolàs ${ }^{\dagger}$ and Michele Redi ${ }^{\ddagger}$ \\ Center for Cosmology and Particle Physics, \\ Department of Physics, New York University \\ 4 Washington Place, New York, NY 10003
}

\begin{abstract}
Motivated by the severity of the bounds on Lorentz violation in the presence of ordinary gravity, we study frameworks in which Lorentz violation does not affect the spacetime geometry. We show that there are at least two inequivalent classes of spontaneous Lorentz breaking that even in the presence of gravity result in Minkowski space. The first one generically corresponds to the condensation of tensor fields with tachyonic mass which in turn is related to ghost-condensation. In the second class, realized by the DGP model or theories of massive gravitons, spontaneous Lorentz breaking is induced by the expectation value of sources. The generalization to de-Sitter space is also discussed.
\end{abstract}

*email: gd23@nyu.edu

†email: pujolas@ccpp.nyu.edu

$\ddagger$ email: redi@physics.nyu.edu 


\section{Introduction}

The possibility of breaking Lorentz invariance is a deep question. In General Relativity, the continuous spacetime symmetries, such as the Lorentz symmetry, are part of gauge invariance, and as such, their breaking can only be understood as the low energy limit of some underlying spontaneous breaking.

Most phenomenological studies in the literature consider explicit breakings, in the limit where gravity is decoupled. The latter however is expected to have very profound consequences, because any order parameter that spontaneously breaks Lorentz invariance will couple to gravity, at least minimally, distorting the geometry of spacetime. Enforcing the cosmological bounds could place such severe constraints on the order parameters, that the corresponding Lorentz violation would have limited observational interest.

This issue can be illustrated considering the simplest possible model of spontaneous Lorentz symmetry breaking, obtained through a time dependent scalar field, $\phi$. Due to the vacuum expectation value $(\mathrm{VEV}), \partial_{0} \phi \neq 0$, the Lorentz symmetry is broken to the rotation subgroup and this breaking will be communicated to ordinary particles (e.g., electrons or photons) by higher dimensional operators of the form,

$$
\frac{1}{\Lambda_{1}^{4}} \partial_{\mu} \phi \partial_{\nu} \phi \bar{\psi}_{e} \gamma^{\mu} \partial^{\nu} \psi_{e}+\frac{1}{\Lambda_{2}^{4}} \partial_{\mu} \phi \partial_{\nu} \phi F_{\alpha}^{\mu} F^{\nu \alpha},
$$

where $\Lambda_{1,2}$ are the scales of new physics. Substituting the non-zero VEV of the scalar time derivative, the above operators generate an effective Lorentz violating dispersion relation for the electron and the photon, which could potentially be observed if the corrections are at least of order $10^{-15}$ and $10^{-23}$ respectively [1].

However, taking into the account gravity, immediately makes the prospect of such observations pretty dim. Indeed, the time dependence of $\phi$ will also contribute to the energy density of the Universe, which should not exceed the critical one because it cannot be cancelled by a readjustment of the cosmological constant or any other conventional source. This implies that at best $\partial_{0} \phi \sim\left(10^{-3} \mathrm{eV}\right)^{2}$. Demanding that the Lorentz violating contributions to the dispersion relation are potentially observable without conflicting with the cosmological bound requires that $\Lambda_{1} \lesssim 10 \mathrm{eV}$ and $\Lambda_{2} \lesssim K e V$ ! Such small values are ruled out by particle physics observations. The situation is a little better when the Lorentz violation affects the mass parameters. For example for the photon, the operator

$$
\frac{1}{\Lambda_{3}^{2}} \partial_{\mu} \phi \partial_{\nu} \phi A^{\mu} A^{\nu}
$$

with $\Lambda_{3}$ as high as $T e V$ [2], would still produce Lorentz violating effects not far from the observable bounds. 
Thus, it is very important to understand the consistent ways of spontaneous breaking of the Lorentz invariance, which even in the presence of gravity do not affect the background geometry. For instance, having Lorentz violation in exact Minkowski or de Sitter spaces. Such theories would provide a consistent underlying framework for the phenomenological studies of Lorentz violations such as [1].

In this note we investigate different scenarios of such Lorentz violation. A large class of Lorentz violating theories which do not change the background corresponds to the condensation of tachyons of arbitrary integer spin fields. We show that these theories in a certain limit of parameter space effectively become equivalent to some type of ghost condensation [3]. The basic feature of these theories is that nowhere in parameter space one can recover a consistent Lorentz invariant vacuum. This is different from the usual breaking of internal symmetries where instead there is a consistent effective field theory description of the unbroken phase. The reason for this is that the instability that breaks Lorentz invariance can only be stabilized by cutoff sensitive physics. While this may cast some shadows on the possibility to find ultraviolet completions of these theories, fluctuations around the Lorentz breaking vacuum can give rise to consistent low energy effective theories.

We point out that there exists a second inequivalent class of theories in which spontaneous Lorentz breaking is accomplished due to the VEV of the energy momentum tensor. This breaking is analogous to the one induced by a cosmological fluid however the space remains Minkowskian and time independent. We show that this possibility is in fact realized in the DGP model [4] and massive gravity theories. Contrary to the previous case there is no strong sensitivity to the cut-off and there is a smooth limit in parameter space where a Lorentz invariant ground state is recovered. Similarly we show that in de-Sitter space it is possible to break the isometries of the space while keeping the space maximally symmetric. In this case however the breaking necessarily dilutes with the expansion of the Universe.

\section{High Spin Tachyons}

A natural way to try to spontaneously break Lorentz invariance in flat space is by following the analogy with the breaking of internal continuous symmetries. For this purpose one needs to introduce a bosonic integer spin order parameter $A_{\mu_{1}, . . \mu_{n}}$ and make it condense. Since $A$ transforms non-trivially under Lorentz, its VEV spontaneously breaks Lorentz invariance. In the presence of gravity the cosmological constant in the action can be chosen so that the ground state remains exactly Minkowski space. In order to achieve this, one needs two ingredients. Schematically, a tachyonic mass term,

$$
-m^{2} A^{2}
$$


and a stabilizing (non-derivative) self-interaction, e.g.,

$$
\lambda A^{4}
$$

The seeming exception is a spin-0 field for which what needs to condense is actually the derivative of a ghost-field [3]. However this difference is only naive. The reason for this is that for higher spin fields the tachyonic and ghost instabilities are inseparable. To illustrate the point let us consider first a massive spin-1 field (see also [5] and references therein),

$$
S=\int d^{4} x\left(-\frac{1}{4} F^{2}-\frac{m^{2}}{2} A^{2}\right)
$$

The gauge invariance of the action can be restored introducing a Stückelberg field that compensates the variation of the gauge field under a gauge transformation. The action becomes,

$$
S=\int d^{4} x\left(-\frac{1}{4} F^{2}-\frac{m^{2}}{2}(\partial \phi+A)^{2}\right)
$$

which is manifestly invariant under,

$$
\begin{aligned}
\delta A_{\mu} & =\partial_{\mu} \epsilon \\
\delta \phi & =-\epsilon .
\end{aligned}
$$

It is clear (we use mostly plus signature) that a tachyonic mass for the gauge field $\left(m^{2}<0\right)$ is associated with a ghost-like kinetic term for the Stückelberg field. This is the tachyonic counterpart of the standard Higgs effect where a massless spin-1 field acquires mass by eating a Goldstone boson with positive kinetic term.

The mechanism outlined above is actually completely general. Take for simplicity a massive spin-2 field (up to some technical modification the same holds for higher spin, see appendix A). The only consistent mass term for the graviton around Minkowski vacuum is described by the Pauli-Fierz (PF) combination,

$$
V=\frac{m^{2} M_{4}^{2}}{8}\left(h_{\mu \nu}^{2}-h^{2}\right),
$$

where $h_{\mu \nu}=g_{\mu \nu}-\eta_{\mu \nu}$. The gauge invariance of the mass term can be restored introducing Stückelberg fields,

$$
h_{\mu \nu}=\hat{h}_{\mu \nu}+\partial_{\mu} A_{\nu}+\partial_{\nu} A_{\mu}
$$

The mass term is then invariant with respect to,

$$
\begin{aligned}
\delta \hat{h}_{\mu \nu} & =\partial_{\mu} \xi_{\nu}+\partial_{\nu} \xi_{\mu} \\
\delta A_{\mu} & =-\xi_{\mu}
\end{aligned}
$$


By plugging (2.7) into the PF mass term one can see that $A_{\mu}$ has the kinetic term of a gauge field. The sign is such that a tachyonic instability for the spin-2 is translated into a ghost instability for the Stückelberg field. Actually $A_{\mu}$ has three degrees of freedom, which can be split into spin-1 and spin-0 polarizations. Since the spin-0 component mixes with $\hat{h}_{\mu \nu}$ it is useful to diagonalize the kinetic terms. This can be achieved through the decomposition,

$$
h_{\mu \nu}=\tilde{h}_{\mu \nu}+\partial_{\mu} \tilde{A}_{\nu}+\partial_{\nu} \tilde{A}_{\mu}+\frac{1}{3 m^{2}} \partial_{\mu} \partial_{\nu} \phi+\frac{1}{6} \eta_{\mu \nu} \phi
$$

With this, the scalar Stückelberg acquires a positive kinetic term even for $m^{2}<0$. This can be understood from the fact that a higher spin field becomes massive by eating a "massive" ghost which in turn can be decomposed into massless ghost and a tachyon Stückelberg with positive kinetic term.

We should here mention that although the mass term for ghost-free theories is uniquely defined, it becomes unclear what form should be used if one wants to create instabilities. The guideline in this case is that if one does not want to increase the number of propagating degrees of freedom, then one has to use the form (2.6) even for the tachyonic case. Of course, such theories can only make sense if the tachyonic instability is stabilized by some non-linear self-interaction 1 . While it is not the purpose of this work to discover the form of such selfinteraction, one natural choice would be to take the stabilizing potential to be a function of the PF combination. Our main point however is that any viable model of spontaneous Lorentz breaking through the condensation of integer spin tachyons, will be accompanied by ghost type instabilities, which have to be stabilized in the true vacuum of the theory, if such exists. As a result these types of theories cannot have a consistent Lorentz invariant vacuum because, in order to recover such a vacuum by continuum change of parameters one has to reverse the sign of the kinetic term of the condensing ghost which requires going through infinite strong coupling. In other words the ghost instabilities are entirely dominated by cut-off sensitive physics.

\section{Lorentz Violation in DGP}

We now turn to the DGP model [4]. This scenario is specified by the action,

$$
S=-\frac{M_{4}^{2}}{2} \int d^{4} x \sqrt{-g} R_{4}-\frac{M_{5}^{3}}{2} \int d^{4} x d y \sqrt{-G} R_{5}
$$

\footnotetext{
${ }^{1}$ In fact, such self interactions could be used to stabilize extra time dimensions [6], since these theories reduce to a tower of spin-2 tachyons (see Appendix A).
} 
which describes a tensionless brane with induced kinetic term for the graviton embedded in a five dimensional empty bulk. (Later, we shall consider the generalization with brane and bulk cosmological constants.)

In DGP there are known static solutions that break Lorentz invariance in Minkowski space 7]. By taking an energy-momentum tensor localized on the brane of the form,

$$
T_{M N}=\rho_{0} \delta(y) \operatorname{diag}(0,-1,-1,-1,0)
$$

it is easy to check that the exact solution for the metric is given by,

$$
d s^{2}=-(1+c|y|)^{2} d t^{2}+d x_{1}^{2}+d x_{2}^{2}+d x_{3}^{2}+d y^{2}
$$

where $c=-\rho_{0} / M_{5}^{3}$. Moreover, in [7] it was shown that the evolution of pressureless dust on a brane with negative tension $\left(\rho_{0}<0\right)$ automatically relaxes to such a ground state.

An important feature of the solution above is that it does not depend on $M_{4}$ so that the DGP kinetic term is only needed to reproduce $4 D$ gravity at distances shorter than the crossover scale $r_{c}=M_{4}^{2} /\left(2 M_{5}^{3}\right)$. Each slice at fixed $y$ is just Minkowski space, however, due to the $y$ dependence, the solution globally breaks Lorentz invariance to the rotation subgroup (in the preferred frame) since the speed of light varies along the fifth dimension. An observer living at fixed $y$ will only detect the Lorentz violation through the non-relativistic dispersion of bulk gravity modes. 2 .

The metric above is actually closely related to the domain-wall solutions studied recently in 8]. In that case it was found that for a domain wall the metric is just the metric of a codimension two object in $5 D$, i.e. it is independent of the DGP kinetic term. This similarity is no accident: the energy momentum tensor (3.2) can be thought as a homogeneous distribution of parallel (euclidean) domain walls. Since for codimension two objects one can find exact solutions for an arbitrary number of defects, the metric (3.3) can directly be obtained from the domain wall one.

In the light of the above one can also construct space-times with different Lorentz breaking patterns in Minkowski space. Consider a distribution of Lorentzian domain walls,

$$
T_{M N}=\rho_{0} \delta(y) \operatorname{diag}(1,-1,-1,0,0)
$$

The metric is then given by,

$$
d s^{2}=-d t^{2}+d x_{1}^{2}+d x_{2}^{2}+(1+c|y|)^{2} d x_{3}^{2}+d y^{2}
$$

${ }^{2}$ Lorentz violation will also be transmitted at tree level to the Standard Model fields if the brane has some thickness in the bulk or by couplings of the the extrinsic curvature to Standard Model bilinears induced at loop level. 
which breaks Lorentz invariance to the subgroup generated by $\left(J_{3}, K_{1}, K_{2}\right)$. Note that for domain walls with positive tension $c<0$, so the requirement that the space is regular at $y=-1 / c$ implies that $x_{3}$ is a periodic coordinate.

\section{Massive Gravity and Other Solutions}

Given the existence of Lorentz violating solutions in DGP it is natural to wonder if they are equivalent to some kind of tachyon condensation. That this is not the case can be already understood from the fact that we can smoothly recover a Lorentz invariant ground state by taking the source to zero. To clarify how this mechanism works from the $4 D$ point of view it is useful to deconstruct the DGP solution of the previous section in terms of massive gravitons. This will also show that this mechanism of Lorentz violation is general to theories of massive gravitons.

To linear order consistent theories of massive gravitons are governed by the PF equation,

$$
\mathcal{E}_{\mu \nu}^{\alpha \beta} h_{\alpha \beta}+\frac{m^{2}}{2}\left(h_{\mu \nu}-\eta_{\mu \nu} h\right)=\frac{T_{\mu \nu}}{M_{4}^{2}},
$$

where $\mathcal{E}_{\mu \nu}^{\alpha \beta}$ is the linearized Einstein tensor,

$$
\mathcal{E}_{\mu \nu}^{\alpha \beta} h_{\alpha \beta}=\frac{1}{2}\left(\partial_{\alpha} \partial_{\nu} h_{\mu}^{\alpha}+\partial_{\alpha} \partial_{\mu} h_{\nu}^{\alpha}-\partial_{\mu} \partial_{\nu} h-\square h_{\mu \nu}-\eta_{\mu \nu} \partial_{\alpha} \partial_{\beta} h^{\alpha \beta}+\eta_{\mu \nu} \square h\right)
$$

In absence of sources the PF mass term guarantees that there are no ghosts or tachyons and the vacuum is Lorentz invariant. Let us now consider a constant energy momentum tensor. The system admits a static solution,

$$
h_{\mu \nu}=-\frac{2}{m^{2} M_{4}^{2}}\left(T_{\mu \nu}-\frac{1}{3} \eta_{\mu \nu} T\right) .
$$

This solution is pure gauge $\left(h_{\mu \nu}=\partial_{\mu} \xi_{\nu}+\partial_{\nu} \xi_{\mu}\right)$ so the space remains flat. However due to this VEV, probe particles will detect a Lorentz breaking background which is static and Minkowskian. Of course whether the space remains flat to non linear order will depend on the interaction terms.

We can now deconstruct the DGP solution. Consider fluctuations of the metric around the vacuum, $g_{\mu \nu}=\eta_{\mu \nu}+h_{\mu \nu}(x, y)$. At the linear level, DGP reduces to a continuum of massive gravitons,

$$
h_{\mu \nu}(x, y)=\int_{0}^{\infty} d m h_{\mu \nu}^{(m)}(x) \psi^{(m)}(y)
$$

where the wave-functions $\psi^{(m)}(y)$ are determined by,

$$
\left(\partial_{y}^{2}+m^{2}+m^{2} r_{c} \delta(y)\right) \psi^{(m)}(y)=0
$$


and each massive graviton satisfies the PF equation,

$$
\left(\mathcal{E} h^{(m)}\right)_{\mu \nu}+\frac{m^{2}}{2}\left(h_{\mu \nu}^{(m)}-\eta_{\mu \nu} h^{(m)}\right)=\frac{1}{M_{5}^{3}} \int d y \psi^{(m)}(y) T_{\mu \nu}(y) .
$$

For the energy-momentum tensor (3.2) one gets,

$$
h_{\mu \nu}^{(m)}=-\frac{2 \rho_{0}}{m^{2} M_{5}^{3}} \psi^{(m)}(0) \operatorname{diag}(1,0,0,0)
$$

from which plugging in eq. (4.4) one can reconstruct the bulk solution (3.3) to linear order.

The linear analysis also suggests that it might be possible to find other Lorentz violating solutions in Minkowski space when $T_{00}$ is not zero. This simply follows from the fact that for any $T_{\mu \nu}$ which is constant the massive graviton has a solution (4.3) which is pure gauge. These solutions can be easily completed to non linear level when the source is a perfect fluid. In general this requires both bulk and brane cosmological constants (the same type of solutions were also discussed in [10] in a different context). To see how this works recall that in the DGP model the induced metric on the brane satisfies

$$
M_{4}^{2} G_{\mu \nu}-2 M_{5}^{3}\left(K_{\mu \nu}-g_{\mu \nu} K\right)=T_{\mu \nu}
$$

where $K_{\mu \nu}$ is the extrinsic curvature on the brane and $T_{\mu \nu}$ the localized energy momentum tensor. Consistency of the bulk equation requires that the Gauss equation is satisfied [11],

$$
G_{\mu \nu}=-\frac{\Lambda_{5}}{2 M_{5}^{3}} g_{\mu \nu}+K K_{\mu \nu}-K_{\mu}^{\rho} K_{\rho \nu}-\frac{1}{2} g_{\mu \nu}\left(K^{2}-K_{\rho \sigma} K^{\rho \sigma}\right)+E_{\mu \nu},
$$

where $E_{\mu \nu}$, the "dark radiation term", is traceless and we have also included a bulk cosmological constant $\Lambda_{5}$. The solution in the bulk generically is Schwarzchild-AdS, $E_{\mu \nu}$ is related to the mass of the bulk black hole (see Appendix B for details). Since we look for a solution where the induced metric is flat we demand,

$$
2 M_{5}^{3}\left(K_{\mu \nu}-\eta_{\mu \nu} K\right)=-T_{\mu \nu}
$$

which implies,

$$
K_{\mu \nu}=-\frac{1}{2 M_{5}^{3}}\left(T_{\mu \nu}-\frac{1}{3} \eta_{\mu \nu} T\right)
$$

The integrability condition (4.9) then determines the cosmological constant and the dark radiation term. For $T_{\nu}^{\mu}=\rho_{0} \operatorname{diag}(-1, w, w, w)$ one obtains

$$
\begin{aligned}
E_{\nu}^{\mu} & =-(w+1) \frac{\rho_{0}^{2}}{8 M_{5}^{6}} \operatorname{diag}\left(-1, \frac{1}{3}, \frac{1}{3}, \frac{1}{3}\right) \\
\Lambda_{5} & =(1+3 w) \frac{\rho_{0}^{2}}{12 M_{5}^{3}} .
\end{aligned}
$$


Note that $\Lambda_{5}$ and the black hole mass are quadratic in perturbation on the brane. As a consequence the energy momentum tensor appearing in (4.6) is only given by the fluid on the brane to linear level. We postpone the construction of the full solution to Appendix B. Here we shall advance that for $w<-1$, the metric takes the form

$$
d s_{5}^{2}=d y^{2}-\frac{\sqrt{C}}{\ell_{A d S}} \frac{\sinh ^{2}\left(2\left(|y|+y_{0}\right) / \ell_{A d S}\right)}{\cosh \left(2\left(|y|+y_{0}\right) / \ell_{A d S}\right)} d T^{2}+\sqrt{C} \ell_{A d S} \cosh \left(2\left(|y|+y_{0}\right) / \ell_{A d S}\right) d x_{3}^{2}
$$

where

$$
\cosh ^{2}\left(2 y_{0} / \ell_{A d S}\right)=-\frac{24}{1+w}\left(\frac{\ell_{A d S} \rho_{0}}{6 M_{5}^{3}}\right)^{2} .
$$

The asymptotic AdS radius is given by $\ell_{A d S}^{2}=-6 M_{5}^{3} / \Lambda_{5}$ and $C$ is an irrelevant scale with units of length ${ }^{2}$.

\subsection{Stability}

An important issue is the stability of the Lorentz violating backgrounds. Let us first address the ghost instabilities. The fact that we have a continuous controllable parameter $\rho_{0}$, guarantees that there is a finite range for which the solutions are ghost free. Indeed, since for $\rho_{0}=0$ there are no ghosts, by continuity this remains true for certain finite range. The minimal size of this range can be estimated from the following argument. For very small $\rho_{0}$, the linearized theory is a good approximation, and is stable. Ghost instabilities, if they ever arise, can only set in when the non-linearities become important. On the other hand, the leading non-linearities come from trilinear interactions of longitudinal gravitons and their relative strength is suppressed by the strong coupling scale $\Lambda_{\text {strong }}^{3}=2 M_{4} / r_{c}^{2}[9]$. This gives the most conservative upper bound on the tension,

$$
\rho_{0}<\frac{M_{4}^{2}}{r_{c}^{2}}
$$

By taking $r_{c}$ of the order of the horizon scale this gives $\rho_{0}<(m e V)^{4}$.

The argument above does not rule out the presence of tachyonic instabilities 3 . These instabilities arise depending on the fluid and are analogous to the ones of Einstein's static Universe. Note however that the time scale of these instabilities, which is under control in the effective description, is set by the source so it can be made arbitrarily large by appropriate choice of parameters (see below).

The tachyon instability can be seen from the Friedman equation, which in DGP with bulk

\footnotetext{
${ }^{3}$ We would like to thank Shinji Mukohyama for discussions about this point
} 
cosmological constant and dark radiation becomes (see Appendix B),

$$
6 \epsilon M_{5}^{3} \frac{\sqrt{f(R)+\dot{R}^{2}}}{R}=-3 M_{4}^{2} \frac{\dot{R}^{2}+\kappa}{R^{2}}+\rho(t),
$$

where $R(t)$ is the scale factor,

$$
f(R)=\kappa-\frac{R^{2}}{\ell^{2}}-\frac{C}{R^{2}},
$$

$\ell^{2}=6 M_{5}^{3} / \Lambda_{5}$ is the de-Sitter radius and $C$ is the mass of the black hole in the bulk. Consider first the case without DGP term. Eq. (4.15) implies,

$$
\dot{R}^{2}-\left(\frac{R^{2}}{\ell^{2}}+\frac{C}{R^{2}}+\frac{R^{2} \rho^{2}}{36 M_{5}^{6}}\right)=-\kappa
$$

This is the equation for a particle with energy $-k / 2$ moving in a potential $V(R)$. From this equation it follows that a negative bulk cosmological constant (corresponding imaginary $\ell$ ) can make the potential positive in some region, and a local minimum with zero potential is in general possible. This is the case for some of the solutions that we found. The inclusion of the DGP term modifies this conclusion in the following way. Any flat space solution found before is obviously a solution with the DGP term. If we consider fluctuations around this background, expanding (4.15) to quadratic order, $R(t)=R_{0}+\delta(t)$, we see that the kinetic term is given by,

$$
\left(\frac{3 \epsilon M_{5}^{3}}{R_{0} \sqrt{f\left(R_{0}\right)}}+\frac{3 M_{4}^{2}}{R_{0}^{2}}\right) \delta^{\prime 2}
$$

Using the equation of motion (4.15) for the solution, one sees that the kinetic term is

$$
3 \frac{6 M_{5}^{6}+\rho_{0} M_{4}^{2}}{R_{0}^{2} \rho_{0}} \delta^{\prime 2}
$$

Therefore, if $\rho_{0}<0$, the DGP term can change the stability of the solutions making certain unstable configurations stable and viceversa. In the general with a cosmological constant $\Lambda_{4}$ and a fluid with energy density $\rho_{0}^{\prime}$ and equation of state $w^{\prime}$ on the brane, then the second derivative of the potential is

$$
-\frac{\left(w^{\prime}+1\right) \rho_{0}^{\prime}\left[\left(3 w^{\prime}-1\right) \Lambda_{4}+2\left(3 w^{\prime}+1\right) \rho_{0}^{\prime}\right]}{6 M_{5}^{6}+\left[\Lambda_{4}+\rho_{0}^{\prime}\right] M_{4}^{2}} .
$$

For a generic fluid there is always a range of parameters for which the solution corresponds to the minimum of the potential and so it is stable. In the case of instabilities, for realistic values of the parameters, the time scale of the instability is of order of Hubble. 


\section{De Sitter Breaking}

In this section we discuss the spontaneous breaking of the isometries of de-Sitter space. Similar arguments could also be applied to Anti-de-Sitter.

At the linear level a massive graviton on de-Sitter space must be described by the PF equation (4.1) where $\mathcal{E}_{\mu \nu}^{\alpha \beta}$ is now the linearized Einstein tensor for de-Sitter space and $\eta_{\mu \nu}$ is replaced by de Sitter metric,

$$
d s^{2}=-d t^{2}+e^{2 H t} d x_{i} d x_{i}
$$

Similarly to the Minkowski case we would like to find solutions which remain de Sitter space but break its isometries due to the VEV of the energy-momentum tensor. Following the flat space example in order to achieve this at linear order one would need to find solutions of the equation,

$$
h_{\mu \nu}-\hat{g}_{\mu \nu} h=-\frac{2 T_{\mu \nu}}{m^{2} M_{4}^{2}}
$$

which are pure gauge on de Sitter, i.e. $h_{\mu \nu}=D_{\mu} \xi_{\nu}+D_{\nu} \xi_{\mu}$. One can easily prove that this is not possible at least for any perfect fluid which is homogenous and isotropic. The crucial point is that due to the cosmological expansion a conserved $T_{\mu \nu}$ is time dependent,

$$
T_{\nu}^{\mu}=\frac{\rho_{0}}{a^{3(1+w)}} \operatorname{diag}(-1, w, w, w)
$$

where $w$ is the equation of state of the fluid and $a=e^{H t}$. Solving eq. (15.2) one finds,

$$
h_{\nu}^{\mu}=\frac{2}{m^{2} M_{4}^{2}} \frac{\rho_{0}}{a^{3(1+w)}} \operatorname{diag}\left(w+\frac{2}{3},-\frac{1}{3},-\frac{1}{3},-\frac{1}{3}\right) .
$$

Plugging this into the linearized Einstein (or Riemann) tensor one can easily check that this is not pure gauge for any choice of $w$. Therefore it is impossible to break Lorentz invariance in de-Sitter space in a theory with a single massive graviton.

The conclusion above can be circumvented in a theory with several massive gravitons. The reason for this is that for a general fluid the solution of the Pauli-Fierz equation on de Sitter is given by,

$$
h_{\nu}^{\mu}=\frac{2}{\left(m^{2}-2 H^{2}\right) M_{4}^{2}} \frac{\rho_{0}}{a^{3(1+w)}} \operatorname{diag}\left(w+\frac{2}{3},-\frac{1}{3},-\frac{1}{3},-\frac{1}{3}\right) .
$$

A curios feature of massive gravity on de-Sitter space is that the only consistent theories must have mass greater than $2 H^{2}$ [12]. It follows that massless and massive gravitons respond with opposite sign to a fluid. Since the metric measured is in general a superpositions of modes as in (4.4), it should be possible to arrange a system so that the total curvature does not change. For example in a theory with extra-dimensions a fluid localized on a de-Sitter brane gives rise to a fluid with the same equation of state for each massive graviton, i.e. $T_{\mu \nu}^{(m)}=T_{\mu \nu}(0) \psi^{(m)}(0)$ 
where $\psi^{(m)}(y)$ is the wave function of the mode $m$. The five-dimensional metric is then to linear order,

$$
h_{\nu}^{\mu}(x, y)=\left(\int \frac{\psi^{(m)}(y) \psi^{(m)}(0)}{2 H^{2}-m^{2}} d m\right) \frac{2 \rho_{0}}{M_{4}^{2} a^{3(1+w)}} \operatorname{diag}\left(w+\frac{2}{3},-\frac{1}{3},-\frac{1}{3},-\frac{1}{3}\right) .
$$

It follows that the curvature at the brane is different from zero unless the integral vanishes due to the cancellation of the zero mode and the contribution of the entire Kaluza-Klein tower.

Remarkably this possibility can be realized in DGP. To set-up a de-Sitter background we take $T_{\mu \nu}=-\Lambda_{4} g_{\mu \nu}$ and a bulk cosmological constant $\Lambda_{5}$. As in Minkowski space to break the isometries we wish to add a non-invariant energy momentum tensor while keeping the induced metric on the brane exactly de-Sitter. From eq. (4.8) we see that this can be achieved by compensating the de-Sitter breaking energy momentum tensor with the extrinsic curvature,

$$
2 M_{5}^{3}\left(K_{\mu \nu}-g_{\mu \nu} K\right)=-T_{\mu \nu}
$$

For a general equation of state we have then,

$$
\begin{aligned}
T_{\nu}^{\mu} & =\frac{\rho_{0}}{a^{3(1+w)}} \operatorname{diag}(-1, w, w, w), \\
K_{\nu}^{\mu} & =\frac{1}{2 M_{5}^{3}} \frac{\rho_{0}}{a^{3(1+w)}} \operatorname{diag}\left(w+\frac{2}{3},-\frac{1}{3},-\frac{1}{3},-\frac{1}{3}\right) \\
E_{\nu}^{\mu} & =\frac{E_{0}}{a^{4}} \operatorname{diag}\left(-1, \frac{1}{3}, \frac{1}{3}, \frac{1}{3}\right),
\end{aligned}
$$

where, since $E_{\nu}^{\mu}$ is traceless and conserved, it decays like radiation. In order to fulfill the Gauss constraints (4.9) the terms quadratic in $K_{\nu}^{\mu}$ should compensate the dark radiation $E_{\nu}^{\mu}$. Given that this scales as $1 / a^{4}$, this requires $w=-1 / 3$. One can then check that Eqs. (4.9) are satisfied by choosing

$$
\Lambda_{4}=r_{c} \Lambda_{5}
$$

and

$$
E_{0}=-\frac{1}{12}\left(\frac{\rho_{0}}{M^{3}}\right)^{2}
$$

The full bulk solution will be presented in Appendix B. The 5D geometry is Schwarzchild-de Sitter. In the absence of the fluid, $\rho_{0}=E_{0}=0$, the bulk is $5 \mathrm{D}$ de Sitter and the brane sits at the maximal slice. This implies that the Hubble rates on the brane and in the bulk coincide, and that the brane becomes "stealth". Indeed, when one chooses the condition (5.9), the induced gravity term completely compensates for $\Lambda_{4}$, and as a result the effective tension vanishes. Once we turn on the Lorentz violating fluid on the brane, then the bulk black hole mass is nonzero and inevitably negative. In order to avoid naked singularities in the bulk, it turns out that the sign of $\rho_{0}$ must also be negative. 
Going back to the linearized solution, despite the presence of sources in the bulk, the problem still reduces to the one of massive gravitons coupled to a perfect fluid. This is because, as can be seen from Eq. (5.10), the sources are second order in the perturbation, and so they can be neglected in the linear analysis. What remains to be shown to establish the consistence of the two solutions is then that the integral (5.6) indeed vanishes. We prove this in Appendix C.

It is interesting to compare the breaking of the de-Sitter isometries discussed above with the symmetry breaking due to the condensation of higher spin fields. Consider for example the spin-1 tachyon. Since the stabilizing potential is a function of $A_{\mu} A^{\mu}$ it is clear that a solution with a constant VEV of $A_{0}$ is not affected by the cosmological expansion. This is possible because the tachyon condensate does not reduce to an ordinary fluid. In the DGP case since the symmetry breaking is induced by a conserved energy momentum tensor on the brane the violations of isometries dilutes with the expansion of the universe.

\section{Conclusions}

In this note we investigated different mechanisms for breaking spontaneously Lorentz invariance or more in general isometries of maximally symmetric spaces. One possibility is the condensation of integer spin tensor fields. This scenario in general implies the presence of ghost instabilities which cannot be controlled in an effective field theory framework around a maximally symmetric background. The second possibility is breaking Lorentz invariance through the VEV of the energy momentum tensor. In ordinary four dimensional theories this would necessarily affect the geometry of space-time leading to practically unobservable Lorentz violating effects once cosmological bounds are enforced. This conclusion does not hold in a theory with extra-dimensions such as DGP or even in a theory with massive gravitons because the space can remain maximally symmetric despite the Lorentz violation.

\section{Acknowledgments}

We would like to thank Nima Arkani-Hamed, Jose Juan Blanco-Pillado and especially Gregory Gabadadze and Shinji Mukohyama for interesting discussions. The work of G. D. and M. R. is supported in part by David and Lucile Packard Foundation Fellowship for Science and Engineering, and by the NSF grant PHY-0245068. O. P. acknowledges support from Departament d'Universitats, Recerca i Societat de la Informació of the Generalitat de Catalunya, under the Beatriu de Pinós Fellowship 2005 BP-A 10131. 


\section{Appendix A: Arbitrary Spins}

In this appendix we extend the results of section 2 to higher spin fields. A massive spin $-n$ field can be decomposed into a massless $\operatorname{spin}-n$ and Stückelberg fields of lower spin. For a spin- $n$ an entire tower of massless spin- $(n-1), \ldots, 0$ Stückelberg fields will be required. The most convenient way to construct consistent theories of high spin massive particles is by starting with the massless theory in one extra-dimension [13]. A massless spin $-n$ field is described by a symmetric tensor $H_{\mu_{1} \ldots \mu_{n}}$ subject to a "double trace" constraint,

$$
H_{\alpha \beta \mu_{5} \ldots \mu_{n}}^{\alpha \beta}=0 .
$$

As for the spin-2, at the quadratic level, there is a unique ghost-free consistent lagrangian,

$$
\begin{aligned}
\mathcal{L}= & -\frac{1}{2}\left(\partial_{M} H_{\ldots}\right)^{2}+\frac{n}{2}\left(\partial_{M} H_{\ldots}^{M}\right)^{2}+\frac{n(n-1)}{2}\left(\partial_{M} H_{L \ldots}^{L}\right)^{2} \\
& +\frac{n(n-1)(n-2)}{8}\left(\partial_{M} H_{L \ldots}^{M L}\right)^{2}+\frac{n(n-1)}{2} H_{L \ldots}^{L}\left(\partial_{M} \partial_{N} H_{\ldots}^{M N}\right)^{2}
\end{aligned}
$$

which is gauge invariant with respect to,

$$
\delta H_{M_{1} \ldots M_{n}}=n \partial_{\left(M_{1}\right.} \epsilon_{\left.M_{2} \ldots M_{n}\right)}
$$

where $\epsilon_{M_{2} \ldots M_{n}}$ is a traceless symmetric tensor and the parenthesis denotes symmetrization of the indexes. The massive theory in one dimension less can be derived by compactifying on a circle of radius $1 / m$ and truncating to the first KK level. One of the advantages of this formulation is that it keeps the gauge invariance of the massive theory manifest. In fact the components of the field with indexes in the internal dimension and harmonic dependence on the extra coordinate are automatically the Stückelberg fields of the theory,

$$
H_{\mu_{1} \ldots \mu_{s} y \ldots y}(x, y)=\phi_{\mu_{1} \ldots \mu_{s}}^{(s)}(x) \cos (m y)
$$

From (6.3) it follows that the massive theory is invariant under,

$$
\delta \phi_{\mu_{1} \ldots \mu_{s}}^{(s)}=s \partial_{\left(\mu_{1}\right.} \epsilon_{\left.\mu_{2} \ldots \mu_{s} y \ldots y\right)}-(n-s) m \epsilon_{\mu_{1} \ldots \mu_{s} y \ldots y}
$$

Roughly speaking the meaning of this transformation is that each $\phi^{(s)}$ is a gauge field with an additional shift symmetry required to to be Goldstone boson for $\phi^{(s+1)}$. Differently from the spin-2 case however not all the Stückelberg fields can be gauged away and some of them act as auxiliary fields (this follows from the constraint that $\epsilon$ must be traceless).

Let us now focus on the highest spin Stückelberg field $\phi_{\mu_{1} \ldots \mu_{n-1}}^{(n-1)}$. By plugging (6.4) into the action (6.2) one can see that this field acquires a positive kinetic term of the form (6.2). Note 
also that no mass term is generated because of the global shift transformation in (6.5). For the lower Stückelberg fields the situation is more complicated in general because the ones that are dynamical acquire kinetic terms from the mixing with higher ones.

The previous derivation applies to the positive $m^{2}$ spin $-n$ theories. The tachyonic case can be derived by starting with two time directions and compactifying one of them. This reverses the sign of all the mass terms. The kinetic term for the first Stückelberg is now ghostlike proving that any higher spin tachyon contains ghost instabilities. The lower Stückelbergs which are dynamical will have alternating positive and ghost kinetic terms.

\section{Appendix B: Exact Solutions}

In this Appendix we present the full solutions including the bulk where the brane is maximally symmetric even though it supports Lorentz violating sources. We shall focus on two cases: in the first one, the brane is flat and the source is a perfect fluid with arbitrary equation of state $w=p / \rho$. The Weyl tensor is non-zero, and the bulk is asymptotically AdS. In the second example, the brane geometry is de Sitter and the source is a fluid with equation of state $w=-1 / 3$. As we shall see, this solution requires a positive bulk cosmological constant $\Lambda_{5}$ and a non-zero Weyl tensor as well.

In order to construct the solution, we note that a maximally symmetric brane is compatible with a bulk whose symmetries are only the isotropy and homogeneity of the 3-dimensional spatial slices, i.e., 5D spherical symmetry (see also the discussion in [10]). Given that in the bulk we have Einstein gravity, the Birkhoff theorem ensures that the bulk takes the form of the Schwarzchild-de Sitter metric [14],

$$
d s_{5}^{2}=-f(R) d T^{2}+\frac{d R^{2}}{f(R)}+R^{2} d \Omega_{\kappa}^{2}
$$

where $f(R)=\kappa-(R / \ell)^{2}-C / R^{2}$, and $d \Omega_{\kappa}^{2}$ denotes the line element of a unit 3 dimensional sphere, flat space or hyperbolic space for $\kappa=1,0$ and -1 respectively. The de Sitter curvature radius is given by

$$
\ell^{2}=\frac{6 M_{5}^{3}}{\Lambda_{5}}
$$

and the projected Weyl tensor [11] (see Eq (5.8)) is related to the 5D Black hole mass parameter $C$ through

$$
E_{0}=\frac{3 C}{R^{4}} .
$$

The brane location can always be parameterized by two functions $R=R(t)$ and $T=T(t)$. 
This introduces a reparameterization freedom, that can be fixed by choosing

$$
f(R) \dot{T}^{2}-\frac{\dot{R}^{2}}{f(R)}=1
$$

In this gauge, the induced metric on the brane is simply

$$
d s_{4}^{2}=-d t^{2}+R^{2}(t) d \Omega_{\kappa}^{2}
$$

The brane trajectory, that is the form of $R(t)$, is obtained from the Israel junction condition (4.8). This reduces to the modified Friedman equation

$$
6 \epsilon M_{5}^{3} \frac{\sqrt{f(R)+\dot{R}^{2}}}{R}=-3 M_{4}^{2} \frac{\dot{R}^{2}+\kappa}{R^{2}}+\rho(t)+\Lambda_{4} .
$$

Here, $\rho(t)$ is the energy density of the fluid on the brane, and $\epsilon= \pm 1$ is the sign of the extrinsic curvature. In our conventions, $\epsilon=1$ means that the bulk is the "interior" of the brane.

\section{B.1 Flat Brane with General Fluid}

In this case, the full solution can be read off from (6.6). In the flat slicing $(\kappa=0)$, the metric has a horizon at $R=\left(C \ell^{2}\right)^{1 / 4}$, where now $\ell$ denotes the AdS radius, $\ell^{2}=-6 M_{5}^{3} / \Lambda_{5}$. The brane sits at a constant "radial" coordinate, $R_{0}$. Its actual value (together with $C$ ) is not determined by the Friedman equation (6.10) or by (4.9), which only determine the ratio $E_{0}=3 C / R_{0}^{4}$. What this means is that we can trade a larger black hole with a brane location closer to it. However, once we specify the distance between the brane and the horizon, everything else is fixed. In terms of the proper coordinate along the bulk the metric takes the form presented in (4.13). In those coordinates, it is apparent that the parameter $C$ is irrelevant, as it can be rescaled away. The constant $y_{0}$ is the distance between the brane and the horizon, which is entirely fixed by $\rho_{0}$ and $w$. Note also that (4.13) explicitly goes to flat space in the $\rho_{0} \rightarrow 0$ limit.

¿From Eq. (4.12), we see that for $w<-1$, the bulk black hole has positive mass, and it also follows that $\Lambda_{5}<0$. Given that the brane is flat, a fluid with this equation of state can be composed of the brane cosmological constant $\Lambda_{4}$ plus some fluid with energy density $\rho_{0}^{\prime}$ and equation of state $w^{\prime}$. For $w^{\prime}>-1$ and $\Lambda_{4}<-\rho_{0}^{\prime}$, one obtains that the equation of state of the superposition is $w<-1$. This also implies that the total energy density on the brane is negative. In Figure 1, we show the conformal diagram corresponding to this case. The brane lies outside the black hole horizon, and the bulk corresponds to the region that includes the asymptotic AdS boundary. 
For $w=-1 / 3$ the metric reduces to the one found by Taub long ago [15],

$$
d s^{2}=(1+c|y|)^{-\frac{2}{3}}\left(-d t^{2}+d y^{2}\right)+(1+c|y|)^{\frac{2}{3}} d x_{3}^{2}
$$

with $c=-\rho_{0} / M_{5}^{3}$. As in the solutions of the next subsection, the energy density of the brane $\rho_{0}$ must be negative in order not to have a naked singularity in the bulk.

\section{B.2 De Sitter Brane}

Let us now turn to the solutions with a de Sitter brane. Since the induced metric is given by (6.9), we will have a de Sitter brane by demanding that $R(t)$ is

$$
R(t)=H^{-1} \begin{cases}\cosh (H t) & \text { for } \kappa=1 \\ e^{H t} & \text { for } \kappa=0 \\ \sinh (H t) & \text { for } \kappa=-1 .\end{cases}
$$

Let us now find out what kind of fluid gives rise to this trajectory. The scale factor solves the equation

$$
\dot{R}^{2}+\kappa=(H R)^{2}
$$

If the brane and bulk expansion rates coincide, $H=1 / \ell$, the extrinsic curvature

$$
\frac{1}{R} \sqrt{f(R)+\dot{R}^{2}}=\frac{\sqrt{-C}}{R^{2}}
$$

contributes to (6.10) like a curvature term. In light of this, the Friedmann equation (6.10) reduces to

$$
6 \epsilon M_{5}^{3} \frac{\sqrt{-C}}{R^{2}}=-3 M_{4}^{2} H^{2}+\Lambda_{4}+\rho .
$$

Hence, it is possible to preserve the de Sitter expansion if $H^{2}=\Lambda_{4} / 3 M_{4}^{2}$ and the fluid redshifts like $1 / R^{2}$, which corresponds to an equation of state $w=-1 / 3$. If the brane and the bulk inflate at different rates, then a de Sitter brane is still in principle possible, but this requires a fluid with a very specific time dependent equation of state. Hence, we shall disregard this possibility. Let us emphasize that the condition $H=1 / \ell$ is equivalent to

$$
\Lambda_{4}=r_{c} \Lambda_{5}
$$

and that in the absence of the fluid (and the bulk black hole) it implies that the brane is effectively stealth: for this choice of $\Lambda_{4}$, the brane can only 'fit' in the equator of the 5D de Sitter, so the extrinsic curvature must vanish. This is compatible with the fact that the brane has nonzero tension thanks to the DGP term, which completely screens it, so that the effective tension is zero. 
It is clear from (6.13) that the bulk black hole mass parameter $C$ can only be negative. This means that there is a naked singularity at $R=0$. Thus, the only regular solution must include the 'exterior' of the brane. This corresponds to negative extrinsic curvature, $\epsilon=-1$. Hence, the fluid must also have $\rho<0$.

It is not apparent in the static coordinates (6.6), but the extension of the Schwarzschildde Sitter metric beyond the cosmological horizon has another naked singularity placed at the opposite pole, as shown in Fig. 1. To avoid it, one can place another brane with the same $\Lambda_{4}$ and with the same kind of fluid with $w=-1 / 3$.

Once $R(t)$ is fixed, $T(t)$ is obtained by Eq. (6.8). After some algebra, one arrives to the following equation for $T(R)$,

$$
\partial_{R} T=\frac{\sqrt{-C}}{R f(R) \sqrt{(H R)^{2}-\kappa}} .
$$

This also tells us that the only possible $\mathrm{dS}$ embedding is with a negative mass particle in the bulk, $C<0$.

Let us briefly comment on the continuation beyond the cosmological horizon. For any $\kappa$, $R(t)$ eventually grows without bound. The static coordinates displayed in (6.6) in general cover a finite range of $R$ bounded by the two roots of $f(R)=0$. In our case $\left(C<0\right.$ and $\left.\Lambda_{5}>0\right)$ there is no inner horizon and the outer horizon is at $R=R_{+}>\ell$. Hence, the brane initially fits inside the horizon but in a finite proper time it crosses it. The continuation of (6.6) is done by replacing $T \rightarrow X+i \pi / 2$ and simply allowing for $R>R_{+}$, so that the metric looks like $d s_{5}^{2}=g(R) d X^{2}-d R^{2} / g(R)+R^{2} d \Omega_{\kappa}^{2}$ with $g(R)=-f(R)>0$. Thus, $R$ becomes the time coordinate and it continues to be the scale factor on the brane. Given that this continuation does not affect the junction condition (6.10), the form of $R(t)$ of course does not change when $R_{+}$is crossed. We can also see that $T$ picks up a constant imaginary part from Eq. (6.14), since the r.h.s. has a simple pole at $R=R_{+}$.

\section{Appendix C: Linearized Analysis in de Sitter}

In this appendix, we complete the linearized analysis of the de Sitter violating solution discussed in Section 5. Recall that for the de Sitter brane solution, the background bulk spacetime is five dimensional de Sitter space,

$$
d s^{2}=a^{2}(y) d s_{4}^{2}+d y^{2}
$$




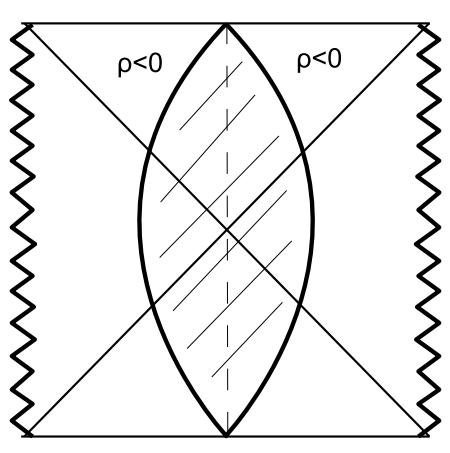

S-dS $(\mathrm{C}<0)$

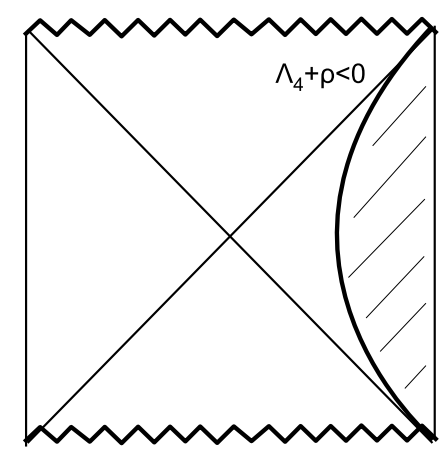

S-AdS

$(\mathrm{C}>0)$

Figure 1: Left: conformal diagrams of the solution with de Sitter branes. The bulk consists of Schwarzschild-de Sitter with negative mass, which has two naked singularities, one at each pole. The static chart displayed in (6.6) covers the interior of the left diamond. In order to avoid the singularity, we have to take $\rho<0$. Still, we have to avoid the other one, which can be accomplished by placing another brane with the same kind of fluid after the equator, represented with the dashed line. Right: conformal diagram for the solution with a flat brane supporting a fluid with generic equation of state. The bulk is Schwarzschild-AdS with positive mass. The effective brane tension is negative, so the solution corresponds to the exterior, including the AdS boundary. The background solution does not depend on $M_{4}$, but gravity is not effectively four dimensional unless the DGP term is included.

with $a(y)=H^{-1} \cos (H y)$. Introducing a Kaluza-Klein decomposition $h_{\mu \nu}=a^{2}(y) \sum_{m} \psi_{m}(y) h_{\mu \nu}^{(m)}(x)$ where the $4 \mathrm{D}$ modes obey (4.1), then one find the following equation for the wave functions,

$$
\left[a^{-4} \partial_{y} a^{4} \partial_{y}+\frac{m^{2}}{a^{2}}+r_{c} m^{2} \delta(y)\right] \psi_{m}(y)=0 .
$$

This equation determines that the spectrum is composed of a massless mode and a continuum of massive gravitons starting at $m^{2}=9 / 4 H^{2}$. Instead of $m$, it is convenient to label each KK level by $p$, defined as

$$
m^{2}=H^{2}\left(p^{2}+9 / 4\right)
$$


Thus, the continuum of KK modes starts at $p=0$, while the zero mode is at $p=3 i / 2$. Plugging the KK decomposition in the equation of motion, we obtain an equation for the wave functions $\psi_{m}(y)$ whose general solution is a linear combination of

$$
U_{i p}(y)=\frac{\Gamma(1-i p)}{\sqrt{2 \pi} 2^{i p} H^{-3 / 2}} \frac{P_{i p}^{3 / 2}(\sin (H y))}{\cos ^{3 / 2}(H y)},
$$

and its conjugate $U_{-i p}$, which are the analogues of the plane waves in de Sitter. Here, $P_{\nu}^{\mu}(z)$ denotes the Legendre function of the first kind and the constants are chosen so that they are normalized to a delta function.

$$
\int_{-\pi / 2 H}^{\pi / 2 H} d y a^{2}(y)\left(1+r_{c} \delta(y)\right) U_{i p}(y) U_{-i p^{\prime}}(y)=\delta\left(p-p^{\prime}\right) .
$$

The $r_{c} \delta(y)$ term in this normalization is required because of the kinetic term localized on the brane (see [16] for a detailed discussion).

The precise combination is dictated by the boundary conditions, which take the form

$$
\partial_{y} \psi-\frac{1}{2} r_{c} H^{2}\left(p^{2}+9 / 4\right) \psi=0
$$

where everything is evaluated at $y=0^{+}$. Hence, KK wave functions are

$$
\psi_{p}^{K K}(y)=\frac{A(-p) U_{i p}-A(p) U_{-i p}}{i \sqrt{2 A(p) A(-p)}}
$$

where

$$
A(p)=U_{i p}^{\prime}(0)-\frac{1}{2} r_{c} H^{2}\left(p^{2}+9 / 4\right) U_{i p}(0) .
$$

The zero mode is normalized as

$$
\int_{-\pi / 2 H}^{\pi / 2 H} d y a^{2}(y)\left(1+r_{c} \delta(y)\right)\left|\psi_{z . m .}(y)\right|^{2}=1 .
$$

Given that $\psi_{z . m .}(y)$ is constant, we obtain

$$
\psi_{z . m .}^{2}=\left(\frac{\pi}{2}+H r_{c}\right) H^{3}
$$

In order to show that to linear order the induced metric remains de Sitter, we need to compute the sum over the spectrum appearing in Eq. (5.6). This can be written as

$$
I=-\frac{1}{2 H^{2}} \psi_{z \cdot m .}(0)^{2}+\frac{1}{H^{2}} \int_{0}^{\infty} d p \frac{\psi_{p}^{K K}(0)^{2}}{p^{2}+1 / 4}
$$


The contribution from the KK modes can be expressed as

$$
\frac{1}{2} \int_{-\infty}^{\infty} d p \frac{1}{p^{2}+1 / 4}\left(U_{i p} U_{-i p}-\frac{A(-p) U_{i p}^{2}}{A(p)}\right)
$$

This can be converted into contour integral where the contour closes the upper half plane. The integral, then, is evaluated by summing over the residues. Given that the Legendre functions have no poles in the $p$ plane, the only poles contributing to (6.16) come from the zeros of $A(p)$ and possibly from $p=i / 2$. It is easy to show that in the upper $p$ plane $A(p)$ vanishes at $p=3 i / 2$. A straightforward computation of the residue shows that this contribution completely cancels that of the zero mode. On the other hand, when the wave functions are evaluated on the brane, at $y=0$, the residue at $p=i / 2$ turns out to be zero. Hence, $I=0$.

\section{References}

[1] S. R. Coleman and S. L. Glashow, "High-energy tests of Lorentz invariance," Phys. Rev. D 59, 116008 (1999) arXiv:hep-ph/9812418.

[2] G. Dvali, M. Papucci and M. D. Schwartz, "Infrared Lorentz violation and slowly instantaneous electricity," Phys. Rev. Lett. 94, 191602 (2005) arXiv:hep-th/0501157.

[3] N. Arkani-Hamed, H. C. Cheng, M. A. Luty and S. Mukohyama, "Ghost condensation and a consistent infrared modification of gravity," JHEP 0405, 074 (2004) arXiv:hep-th/0312099.

[4] G. R. Dvali, G. Gabadadze and M. Porrati, "4D gravity on a brane in 5D Minkowski space," Phys. Lett. B 485, 208 (2000) arXiv:hep-th/0005016.

[5] H. C. Cheng, M. A. Luty, S. Mukohyama and J. Thaler, "Spontaneous Lorentz breaking at high energies," JHEP 0605, 076 (2006) [arXiv:hep-th/0603010].

[6] G. Dvali, G. Gabadadze and G. Senjanovic, 1999 unpublished. See also G. Gabadadze, Proceedings of "Cosmo 99", Trieste, Italy, 1999.

[7] C. Deffayet, G. R. Dvali, G. Gabadadze and A. Lue, "Braneworld flattening by a cosmological constant," Phys. Rev. D 64, 104002 (2001) arXiv:hep-th/0104201].

[8] G. Dvali, G. Gabadadze, O. Pujolas and R. Rahman, "Domain walls as probes of gravity," arXiv:hep-th/0612016. 
[9] C. Deffayet, G. R. Dvali, G. Gabadadze and A. I. Vainshtein, "Nonperturbative continuity in graviton mass versus perturbative discontinuity," Phys. Rev. D 65, 044026 (2002) arXiv:hep-th/0106001]; M. A. Luty, M. Porrati and R. Rattazzi, "Strong interactions and stability in the DGP model," JHEP 0309, 029 (2003) arXiv:hep-th/0303116.

[10] C. Csaki, J. Erlich and C. Grojean, "Gravitational Lorentz violations and adjustment of the cosmological constant in asymmetrically warped spacetimes," Nucl. Phys. B 604, 312 (2001) arXiv:hep-th/0012143.

[11] T. Shiromizu, K. i. Maeda and M. Sasaki, Phys. Rev. D 62, 024012 (2000) arXiv:gr-qc/9910076].

[12] A. Higuchi, "Forbidden mass range for spin-2 field theory in de Sitter space-time," Nucl. Phys. B 282, 397 (1987).

[13] M. Bianchi, P. J. Heslop and F. Riccioni, "More on la grande bouffe," JHEP 0508, 088 (2005) arXiv:hep-th/0504156.

[14] P. Bowcock, C. Charmousis and R. Gregory, "General brane cosmologies and their global spacetime structure," Class. Quant. Grav. 17 (2000) 4745 [arXiv:hep-th/0007177].

[15] A. H. Taub, "Plane symmetric spacetimes," Phys. Rev. 103, 454 (1956).

[16] O. Pujolas, "Quantum fluctuations in the DGP model and the size of the cross-over scale," JCAP 0610, 004 (2006) arXiv:hep-th/0605257. 